\title{
QUEIXAS AUDITIVAS DE DISC JOCKEYS DA CIDADE DE RECIFE
}

\section{Auditory complaints in disc jockeys in Recife}

\author{
Eliza Maia de Britto Macedo ${ }^{(1)}$, Wagner Teobaldo Lopes de Andrade (2)
}

\begin{abstract}
RESUMO
Objetivo: investigar a ocorrência de queixas auditivas em disc jockeys da cidade de Recife/PE. Método: foi realizada uma entrevista com 30 disc jockeys, com idade entre 19 e 28 anos, abordando informações ocupacionais, conhecimentos gerais sobre o ruído e queixas auditivas (diminuição da acuidade auditiva, desconforto a sons intensos, zumbido, sensação de ouvido abafado e otalgia). A análise foi realizada por meio de abordagem quantitativa, utilizando o teste estatístico $t$-student. Resultados: dentre os dados mais relevantes, destacam-se: $46,7 \%$ dos disc jockeys apresentaram, espontaneamente, queixas auditivas, em especial, a diminuição da acuidade auditiva (relatada por todos os sujeitos); 14 disc jockeys (46,67\%) referiram desconforto a sons intensos e $13(43,33 \%)$ mencionaram zumbido. Todos afirmaram ter conhecimento sobre os riscos do ruído para a saúde auditiva, mas $76,7 \%$ não realizam qualquer medida preventiva de suas consequências. A perda auditiva foi referida pelos sujeitos como o principal risco da exposição a níveis intensos de pressão sonora. Conclusão: todos os disc jockeys apresentaram queixa de perda auditiva e, entre as demais queixas auditivas, destacaram-se o desconforto a sons intensos e o zumbido. Tendo em vista a irreversibilidade da perda auditiva induzida por elevados níveis de pressão sonora, os disc jockeys devem ser periodicamente avaliados a fim de que se confirme ou não a perda auditiva de que se queixaram e, caso ela exista, deve ser monitorada para que seja passível de intervenção pelo fonoaudiólogo. Desta forma, percebe-se a necessidade de atuação da Fonoaudiologia junto aos disc jockeys, uma vez que poder-se-á propiciar a otimização do exercício profissional com o mínimo de risco possível.
\end{abstract}

DESCRITORES: Perda Auditiva; Efeitos do Ruído; Ruído Ocupacional

\section{INTRODUÇÃO}

Com o desenvolvimento científico e a evolução tecnológica do mundo moderno, aumentaram consideravelmente os níveis de ruído aos quais o homem passou a estar frequentemente exposto nas ruas, no trabalho e no lazer. Os efeitos nocivos gerados por este ruído, independentemente de suas características específicas, variam principalmente em relação ao nível e frequência de exposição, nível de pressão sonora e duração.

(1) Fonoaudióloga pela Universidade Federal de Pernambuco - UFPE; Graduanda em Medicina pela Faculdade Boa Viagem (FBV), Recife, Pernambuco.

(2) Fonoaudiólogo; Doutorando em Linguística pela Universidade Federal da Paraíba - UFPB; Professor do Departamento de Fonoaudiologia da Universidade Federal da Paraíba - UFPB; Especialista em Audiologia pela Universidade Católica de Pernambuco - UNICAP.

Conflito de interesses: inexistente
Do ponto de vista físico, o ruído é tido como a superposição de vários movimentos de vibrações com frequências e intensidades diferentes e com componentes não harmônicos entre si. Com frequência, a sensação auditiva desencadeada por ele é desagradável especialmente quando é apresentada de modo intenso. Existem sons que são compostos por vibrações cujas frequências harmonizam-se de maneira a produzir música que, do ponto de vista físico, não é considerado ruído'.

O ruído tem sido considerado o mais frequente entre os agentes nocivos à saúde nos ambientes de trabalho e apesar de estar se tornando um problema crescente, o reconhecimento da PAINPSE não é universal. Ela é, ainda, caracterizada como o fator mais prevalente na origem de doenças ocupacionais.

$\mathrm{Na}$ atualidade, a tecnologia se presentifica cada vez mais em todos os setores da vida moderna e, com a evolução da eletrônica, a potência dos 
instrumentos musicais e dos equipamentos de som aumentou de modo significativo. A intensidade com que as músicas são executadas em determinados ambientes pode afetar consideravelmente o sistema auditivo. A intensidade sonora dos ambientes que oferecem a música amplificada como atração chega a ultrapassar bastante os limites de risco para audição humana que, segundo a NR $15^{2}$, é de $85 \mathrm{~dB}$, com exposição de 8 horas diárias.

A música eletrônica amplificada é uma parte bastante importante da programação de vários eventos, incluindo as boates, onde se observa a participação indispensável do disc jockey (DJ). Esse profissional é responsável por misturar/ sobrepor músicas diferentes para serem ouvidas e/ ou dançadas, usando suportes como vinil, compact disc (CD) ou arquivos digitais sonoros, um processo denominado mixagem. Nos últimos anos, esse profissional tem estado bastante susceptível a desenvolver alterações auditivas devido à exposição frequente a níveis de pressão sonora elevados ${ }^{3}$.

São muitas as consequências que os níveis de pressão sonora elevados podem provocar no organismo, dentre elas, o que se conhece como Perda Auditiva Induzida por Níveis de Pressão Sonora Elevados (PAINPSE). A PAINPSE é um distúrbio que cada vez mais vem atingindo a população tanto física como mentalmente, seja em indústrias, usinas, consultórios odontológicos, metalúrgicas, serralharias, boates, casas de shows e outros locais ${ }^{4}$.

Pelo fato de estar exposto por muitas horas a cada noite a sons de alta intensidade, o DJ está sujeito ao surgimento da PAINPSE. Apesar de a característica primordial da PAINPSE ser a diminuição da acuidade auditiva, podem estar presentes outros sintomas como zumbido, desconforto a sons de alta intensidade, sensação de ouvido abafado e dores frente à presença de determinados tipos de ruído de alta intensidade ${ }^{4}$.

Sabe-se do grande número de publicações e estudos científicos que abordam a PAINPSE em trabalhadores de usinas e indústrias, em contrapartida, poucos focalizam alterações auditivas em músicos e DJs que, em sua ocupação, seja profissionalmente ou por hobby, também estão expostos à elevada intensidade sonora.

Desta forma, o objetivo do presente estudo foi investigar a ocorrência de queixas auditivas (perda auditiva, zumbido, desconforto a sons intensos, sensação de ouvido abafado e otalgia) em disc jockeys da cidade de Recife/PE, no ano de 2007.

\section{MÉTODO}

A coleta dos dados foi realizada na cidade de Recife/PE, que apresenta uma área de $217 \mathrm{~km}^{2} \mathrm{e}$ uma população de 1.533 .580 mil pessoas, segundo o IBGE (2007).
A população do estudo foi composta por 30 DJs da cidade de Recife/PE, do sexo masculino, com idade entre 19 e 48 anos.

Foram excluídos os indivíduos que tinham feito ou estivessem fazendo uso de medicamentos ototóxicos, que apresentaram história de problemas auditivos na família, apresentaram distúrbio otológico, que tiveram menos de um ano atuando na profissão ou apresentaram histórico de atividade laborativa com exposição a ruído ocupacional intenso anteriormente à atividade como DJ.

A coleta dos dados ocorreu no período de abril a junho de 2007. O delineamento do estudo foi observacional, descritivo e transversal.

As variáveis independentes de análise foram: idade, tempo em que atua na profissão (quantidade de anos em que trabalha como DJ até o dia de aplicação do questionário), jornada semanal de trabalho (quantidade de dias em que atua como DJ, no período de uma semana) e jornada diária de trabalho (tempo em horas que atua como DJ no período de um dia). Já as variáveis dependentes consideradas foram: queixa de perda auditiva, desconforto a sons intensos, zumbido, otalgia e sensação de ouvido abafado, além da realização de medidas preventivas dos efeitos do ruído (as medidas adotadas pelos participantes para evitar os efeitos advindos da exposição ao ruído).

Os participantes foram contactados pelos pesquisadores, por meio de telefone, quando solicitados a participar da pesquisa. Os que consentiram a participação foram questionados sobre os critérios de exclusão da pesquisa e, com os que não apresentaram qualquer um dos critérios de exclusão, foi agendado um encontro para a realização de uma entrevista. No dia marcado com o participante, anteriormente à entrevista, foi fornecida explicação verbal a respeito do estudo e, em seguida, procedeu-se a leitura e solicitação de assinatura de duas vias do termo de consentimento livre e esclarecido (TCLE) caso concordasse com seu conteúdo. Todos os participantes receberam uma cópia do TCLE.

Posteriormente, os sujeitos foram solicitados a responder a uma entrevista elaborada pelos pesquisadores composta por 10 perguntas semiestruturadas sobre suas informações ocupacionais, conhecimentos sobre o ruído e queixas auditivas de perda auditiva, desconforto a sons intensos, zumbido, sensação de ouvido abafado e otalgia (Figura 1). O procedimento da entrevista foi devidamente treinado e realizado apenas pelos pesquisadores para que a aquisição das respostas ocorresse de forma homogênea. Ao final da entrevista, todos os participantes receberam orientações sobre a preservação da audição. 


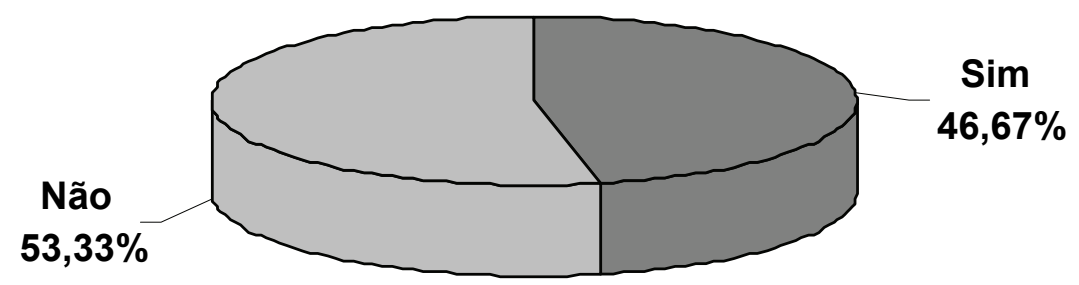

Figura 1 - Distribuição dos sujeitos segundo a referência espontânea de queixa auditiva relacionada ao ruído (Recife, 2007)

Este estudo foi aprovado pelo Comitê de Ética em Pesquisa com Seres Humanos do Centro de Ciências da Saúde da Universidade Federal de Pernambuco (UFPE) sob número 023/2007.

Os dados foram analisados por meio de frequência absoluta e relativa em figuras e tabelas, utilizando o software EPI-INFO for Windows.

\section{RESULTADOS}

A idade dos entrevistados variou de 19 a 48 anos, com média de 32,40 anos, desvio padrão de 8,05 anos e coeficiente de variação de $24,83 \%$ (Tabela 1).

A média de tempo de atuação foi 13,97 anos, com média do tempo de exposição de 5,63 horas diárias, durante aproximadamente quatro dias por semana (Tabela 1).

Do total de participantes, 14 (46,67\%) referiram, espontaneamente, alguma queixa relacionada ao ruído (Figura 2). Quando questionados, especificamente, sobre a sensação de perda auditiva, todos os sujeitos responderam afirmativamente. Todos os pesquisados afirmaram ter conhecimento sobre os riscos do ruído para a saúde geral e referiram a perda auditiva como risco principal.
Além disso, 14 DJs (46,67\%) referiram desconforto a sons intensos, $13(43,33 \%)$ mencionaram zumbido, oito sujeitos $(26,67 \%)$ afirmaram apresentar sensação de ouvido abafado e dois $(6,67 \%)$ referiram otalgia (Tabela 2). Não foi verificada relação estatisticamente significante entre estas queixas auditivas e o tempo de atuação como DJ.

A realização de medidas preventivas dos efeitos do ruído foi referida por 21 DJs (70\%). Das medidas relatadas pelos participantes, destacouse a menção ao uso do protetor auricular no local ruidoso enquanto não está trabalhando (13 sujeitos - 43,33\%), a utilização de estrutura de som de qualidade (3 sujeitos - 10\%), a execução do trabalho em cabina de som com isolamento acústico, a realização de repouso auditivo e a diminuição da intensidade do headfone e do retorno (todas referidas por 2 sujeitos $-6,67 \%)$. Nenhum dos 30 DJs entrevistados referiu usar o protetor auricular durante a atuação profissional.

\section{DISCUSSÃO}

Em geral, o nível de pressão sonora das boates e casas noturnas é bastante alto. Um estudo realizado em casas noturnas de Edinburgh (Reino

Tabela 1 - Estatística descritiva para as variáveis: idade, tempo de atuação como DJ, tempo de exposição semanal e tempo de exposição diária ao ruído (Recife, 2007)

\begin{tabular}{|c|c|c|c|c|c|}
\hline \multirow[b]{2}{*}{ Variável } & \multicolumn{5}{|c|}{ Estatísticas } \\
\hline & Média & $\begin{array}{l}\text { Desvio } \\
\text { padrão }\end{array}$ & $\begin{array}{l}\text { Coeficiente } \\
\text { de variação }\end{array}$ & Mínimo & Máximo \\
\hline - Idade & 32,40 & 8,05 & 24,83 & 19 & 48 \\
\hline $\begin{array}{l}\text { - Tempo de atuação como DJ } \\
\text { (em anos) }\end{array}$ & 13,97 & 9,19 & 65,80 & 1 & 32 \\
\hline $\begin{array}{l}\text { - Tempo de exposição } \\
\text { semanal (em dias) }\end{array}$ & 4,07 & 1,76 & 43,29 & 2 & 7 \\
\hline $\begin{array}{l}\text { - Tempo de exposição diária } \\
\text { (em horas) }\end{array}$ & 5,63 & 2,83 & 50,31 & 1 & 15 \\
\hline
\end{tabular}




\section{ENTREVISTA COM OS PARTICIPANTES}

\section{DADOS DE IDENTIFICAÇÃO}

Nome:

Idade:

Telefone:

Sexo: ( ) M ( ) F Email:

Local de trabalho:

Apresenta alguma queixa relacionada à exposição ao ruído?

\section{INFORMAÇÕES OCUPACIONAIS}

1)Tempo de atuação como DJ

2) Uso de protetor auricular durante o trabalho ( ) Sim ( ) Sistemático ( ) Não

3) Tempo de exposição diária (em horas) e semanal (em dias) ao ruído: I

\section{CONHECIMENTOS GERAIS SOBRE O RUÍDO}

4) Tem conhecimento sobre os riscos do ruído para a saúde geral?

( ) Sim ( ) Não

Quais riscos?

5) Faz uso de alguma medida preventiva contra o ruído?

( ) Sim ( ) Não

Quais?

\section{QUEIXAS AUDITIVAS}

Apresenta:

6) Perda auditiva? ( ) Sim ( ) Não

Obs.:

7) Zumbido? ( ) Sim （ ) Não

Obs.:

8) Dor no ouvido? ( ) Sim ( ) Não

Obs.:

9) Sensação de ouvido abafado? ( ) Sim （） Não

Obs.:

10) Desconforto a sons de alta intensidade? ( ) Sim ( ) Não

Obs.:

Figura 2 - Entrevista realizada com os participantes 
Tabela 2 - Distribuição dos sujeitos segundo a ocorrência das queixas auditivas de desconforto a sons intensos, zumbido, sensação de ouvido abafado e otalgia (Recife, 2007)

\begin{tabular}{|c|c|c|c|c|c|}
\hline Tipo de queixa auditiva & $\mathbf{N}$ & $\%$ & $\begin{array}{l}\text { Média de tempo } \\
\text { (anos) de atuação }\end{array}$ & $\begin{array}{l}\text { Desvio } \\
\text { padrão }\end{array}$ & Valor de $p$ \\
\hline \multicolumn{6}{|c|}{ - Desconforto a sons de alta intensidade } \\
\hline Sim & 14 & 46,67 & 12,14 & 7,12 & $p^{(1)}=0,3176$ \\
\hline Não & 16 & 53,33 & 15,56 & 10,65 & \\
\hline TOTAL & 30 & 100,0 & & & \\
\hline \multicolumn{6}{|l|}{ - Zumbido } \\
\hline Sim & 13 & 43,33 & 13,23 & 9,27 & $p^{(1)}=0,7084$ \\
\hline Não & 17 & 56,67 & 14,53 & 9,37 & \\
\hline TOTAL & 30 & 100,0 & & & \\
\hline \multicolumn{6}{|c|}{ - Sensação de ouvido abafado } \\
\hline Sim & 8 & 26,67 & 9,75 & 8,51 & $p^{(1)}=0,1319$ \\
\hline Não & 22 & 73,33 & 15,50 & 9,12 & \\
\hline TOTAL & 30 & 100,0 & & & \\
\hline \multicolumn{6}{|l|}{ - Otalgia } \\
\hline Sim & 2 & 6,67 & 4,00 & 2,83 & $* *$ \\
\hline Não & 28 & 93,33 & 14,68 & 9,08 & \\
\hline TOTAL & 30 & 100,0 & & & \\
\hline
\end{tabular}

$\left(^{* *}\right)$ - Não foi determinado devido à ocorrência de frequência muito baixa em uma das categorias.

(1) - Por meio do teste t-Student de variâncias iguais.

Unido) ${ }^{5}$ mostrou que os níveis de pressão sonora nestes estabelecimentos variaram entre $97,8 \mathrm{~dB}$ e $107,9 \mathrm{~dB}$. Vale salientar que, de acordo com a legislação brasileira ${ }^{2}, 85 \mathrm{~dB}$ é suficiente para iniciar um processo de diminuição auditiva, caso a exposição se dê por período de tempo superior a 8 horas diárias.

$\mathrm{Na}$ pesquisa realizada em Edinburgh, foi verificado que $13 \%$ dos DJs participantes do estudo possuíam curvas audiométricas características de PAINPSE ${ }^{5}$. Sendo assim, estes podem estar sujeitos ao desenvolvimento ou agravamento de consequências advindas dessa exposição.

$\mathrm{Na}$ presente pesquisa, a população estudada apresentou exposição à música eletronicamente amplificada frequente ao longo da semana, além do que, muitas vezes, não houve um período satisfatório de repouso auditivo, que, segundo a Portaria $19^{6}$, deve ser de 14 horas.

Sabe-se também, que, nos primeiros dez anos de exposição a elevados níveis de pressão sonora, há uma rápida evolução da perda auditiva e, com o passar dos anos, essa progressão se torna mais lenta $^{6}$. Desta forma, diante da média do tempo de atuação como DJ (14 anos) encontrada neste estudo, pode-se supor que há uma grande possibilidade de instalação de uma perda auditiva com tendência à progressão devido à falta de medidas preventivas.

Considerando as médias das variáveis independentes, ter-se-ia, como perfil, um DJ que atua há quase 14 anos, em quatro dias por semana e por mais de 5 horas diárias. Estes são dados que mostram o quão susceptível este sujeito se encontra aos efeitos auditivos decorrentes do ruído.

Estudos tem sistematicamente relatado a ocorrência de perda auditiva em populações expostas ao ruído (ocupacional e não-ocupacional). Entre os estudos mais recentes sobre o tema, encontra-se referência a perda auditiva em $63 \%^{7}$ e $74 \%^{8}$ dos trabalhadores.

Uma pesquisa com músicos de rock and rolp mostrou que estes sujeitos, apesar de não apresentarem perda auditiva diagnosticada à audiometria, já possuem uma alteração coclear percebida às emissões otoacústicas (EOA), mostrando que apesar de a audiometria ainda não revelar perda auditiva, estes sujeitos já apresentam uma lesão coclear. Outros estudos revelam a ocorrência de mudança temporária de limiar (MTL) entre músicos ${ }^{10}$ e DJs ${ }^{11}$ expostos a intensidades elevadas de intensidade sonora.

O percentual de ocorrência de desconforto a sons intensos encontrado nos DJs participantes da presente pesquisa corrobora autores que 
afirmam que, mesmo o individuo que não escuta estímulos fracos, pode chegar a apresentar maior sensibilidade para sons muito intensos, o que torna bastante incômoda a percepção desses estímulos ${ }^{12}$. O desconforto a sons intensos é uma manifestação inerente a uma lesão coclear, sendo assim, é comum que sujeitos expostos a elevados níveis de pressão sonora venham a ter algum tipo de diminuição da acuidade auditiva, acompanhada desta queixa.

A alta ocorrência da queixa de desconforto a sons intensos foi semelhante à de um estudo que revela sua presença em $48 \%$ de músicos de rock and roll ${ }^{9}$ e corrobora estudos que verificaram sua grande ocorrência em sujeitos envolvidos com atividades musicais em alta intensidade ${ }^{13}$.

Com relação ao zumbido, os resultados deste estudo foram discordantes de um estudo realizado com DJs, que verificou que $74 \%$ dos participantes referiram queixa de zumbido ${ }^{5}$, um percentual bastante superior ao encontrado na presente pesquisa. Outro estudo revelou ocorrência de 39\% desta queixa entre músicos de rock and rolp, o que concorda com estudos a respeito da atividade musical em forte intensidade ${ }^{13}$. Outros estudos envolvendo profissionais que exercem atividades musicais revelam ocorrência de zumbido em 67\% de músicos de rock $^{10}$ e em $75 \%$ de DJs ${ }^{14}$. Estudos atuais ${ }^{15-16}$ tem revelado correlação significativa entre a periodicidade do zumbido e o nível do ruído.

Ocasionalmente, pode-se encontrar referência a uma sensação de dor (otalgia) frente à presença de determinados tipos de ruído ${ }^{12}$. No entanto, nesta pesquisa, não foi comum a referência a esta queixa em indivíduos expostos a elevados níveis de pressão sonora.

Apesar dos poucos relatos de sensação de ouvido abafado na presente pesquisa, sabe-se da possibilidade de sua ocorrência. Em um estudo realizado com músicos de frevo e maracatu ${ }^{17}$, foi constatado que pouco menos da metade dos participantes referiram essa queixa. Outro estudo revelou ocorrência de $22 \%$ desta queixa entre músicos de rock and roll $^{9}$, percentual semelhante ao verificado na população de DJs estudada nesta pesquisa.

De forma geral, percebe-se que os DJs são um grupo de risco para apresentar queixas e alterações auditivas ${ }^{10}$, tendo em vista que a exposição ao ruído em casas noturnas frequentemente excede níveis seguros $^{5}$. Além disso, pode-se perceber que, de forma geral, os DJs se mostraram conscientes do risco e conhecem algumas medidas preventivas que, no entanto, da forma como propõem, não se mostra eficaz.
Desta forma, tendo em vista a população estudada, discorda-se da afirmação de que músicos nem sempre estão informados e conscientes do risco da música em forte intensidade para a saúde ${ }^{18}$.

Estudos $^{19-20}$ têm sistematicamente relevado a importância de medidas educativas e de treinamento a fim de minimizar os efeitos do ruído na audição. Outros estudos ${ }^{21-23}$ têm mostrado, ainda, a necessidade de legislação específica aos profissionais ligados à música.

Apesar de a perda auditiva decorrente da exposição ao ruído ser extremamente bem definida, sabe-se que fatores individuais, como $\mathrm{sexO}^{24}$, consumo de cigarro ${ }^{25-26}$, nível de colesterol25-26, pressão sanguínea ${ }^{25}$ e idade ${ }^{27}$ estão diretamente relacionados a esta condição.

Além disso, mesmo sabendo que o ambiente de trabalho dos DJs apresenta razoável vibração, em função da alta intensidade da música em um ambiente fechado, não se pode ainda afirmar que esta vibração seja um agravante do risco para perda auditiva nos disc-jockeys.

\section{CONCLUSÃO}

A exposição prolongada a elevados níveis de pressão sonora sempre significou um risco à saúde auditiva dos trabalhadores. Dentre eles, inclui-se o DJ, uma classe que, assim como tantas outras, pode desenvolver prejuízos à saúde causados por seu instrumento de trabalho, no caso, a música eletronicamente amplificada.

Sabe-se que o aparecimento de alterações auditivas é pertinente a esse tipo de atividade laborativa. Sendo assim, salienta-se que esses trabalhadores estão sujeitos e vulneráveis ao surgimento de tais alterações.

Na população estudada, foi encontrada queixa de alteração auditiva em todos os DJs. Entre as demais queixas auditivas, ressalta-se a ocorrência de desconforto a sons intensos e zumbido.

Entre as sugestões viáveis e eficazes para minimização dos riscos do ruído para a saúde, segundo os DJs, destacaram-se: a utilização de estrutura de som de qualidade, execução do trabalho em cabina de som com isolamento acústico e realização de repouso auditivo.

Neste panorama, a Fonoaudiologia e outras áreas da saúde relacionadas ao exercício profissional devem estar atentas aos riscos sofridos no ambiente de trabalho, objetivando a minimização dos riscos para saúde dos trabalhadores. 


\begin{abstract}
Purpose: to investigate the occurrence of auditory complaints in disc jockeys from the city of Recife/ PE. Method: an interview was carried through with 30 disc jockeys aged between 19 and 48 years, involving occupational information, general knowledge about noise and auditory complaints (diminished auditory acuity, discomfort under intense sound, tinnitus, sultry ear sensation and otalgia). The analysis was carried out through a quantitative approach and using t-student statistical test. Results: among the most relevant data we can empathize that: $46.7 \%$ of the disc jockeys showed, spontaneously, auditory complaints, in special, reduction of the auditory acuity (referred by all subjects); 14 disc jockeys $(46.67 \%)$ related to feel discomfort to intense sound and $13(43.33 \%)$ mentioned tinnitus. All of them affirmed to know the risks of noise related to auditory health, but $76.7 \%$ do not apply any prevention against its consequences. Hearing loss was related as the main risk of exposure to high level of sound pressure. Conclusions: all disc jockeys complained on hearing loss and, among the other auditory complaints, we may emphasize discomfort under intense sound and tinnitus. Considering the irreversibility of high sound pressure induced hearing loss, the disc jockeys might be periodically evaluated as for hearing in order to confirm or discard the hearing loss they complaint. If hearing loss exist, it might be monitored, in order to make possible an intervention by the speech-language therapist. Such being the case, we may perceive the need for speech therapy concerning the disc jockeys for optimizing their professional performance with less risks.
\end{abstract}

KEYWORDS: Hearing Loss; Noise Effects; Occupational Noise

\section{REFERÊNCIAS}

1. Russo ICP. Acústica e Psicoacústica aplicadas à Fonoaudiologia. São Paulo: Lovise; 1999.

2. Brasil. Ministério do Trabalho. Norma regulamentadora $\mathrm{n}^{\circ}$ 15. Brasília, 1978. Disponível em <http:// www.mte.gov.br/legislacao/normas_regulamentadoras/nr_15.pdf>. Acesso em: 28 out. 2009.

3. Jorge Junior JJ, Alegre ACM, Greco MC, Angelini MCA, Barros PM. Hábitos e limiares auditivos de jovens em relação à música eletronicamente amplificada em discotecas. Revista Brasileira de Otorrinolaringologia. 2001;67(3):297-304.

4. Bernardi APA. Testes utilizados na avaliação de trabalhadores expostos a níveis de pressão sonora elevados e solventes. In: Bernardi APA, org. Conhecimentos essenciais para atuar em empresas: Audiologia Ocupacional. São José dos Campos: Pulso; 2003. p. 67-80.

5. Bray A, Szymanski M, Mills R. Noise induced hearing loss in dance music disc jockeys and an examination of sound levels in nightclubs. The Journal of Laryngology \& Otology. 2004;118(2):123-8.

6. Brasil. Ministério do Trabalho. Portaria 19. Brasília, 1998. Disponível em: http://www.mtb.gov.br/temas/ segsau/legislação/portaria/1998/conteudo/port19. asp. Acesso em 28 out. 2009.

7. Dias A, Cordeiro R, Corrente JE, Gonçalves CGO. Associação entre perda auditiva induzida pelo ruído e zumbidos. Cad Saude Publica. 2006;22(1):63-8.
8. Ogido R, Costa EA, Machado HC. Prevalência de sintomas auditivos e vestibulares em trabalhadores expostos a ruído ocupacional. Revista de Saúde Pública. 2009;43(2):377-80.

9. Maia JRF, Russo ICP. Estudo da audição dos músicos de rock and roll. Pró-Fono Revista de Atualização Científica. 2008;20(1):49-54.

10. Pfeiffer M, Rocha LRO, Oliveira FR, Frota S. Intercorrência audiológica em músicos após um show de rock. Revista CEFAC. 2007;9(3):423-9.

11. Santos L, Morata TC, Jacob LC, Albizu E, Marques JM, Paini M. Music exposure and audiological findings in Brazilian disc jockeys (DJs). International Journal of Audiology. 2007;46(5):223-31.

12. Ibañez RN, Schneider LO, Seligman J. Anamnese dos trabalhadores expostos ao ruído. In: Nudelmann AA, Costa EA, Seligman J, Ibañez RN, org. PAIR: Perda auditiva induzida por ruído. vol. III. São Paulo: Revinter; 2001. p. 45-50.

13. Schmuziger N, Patscheke J, Probst R. Hearing in nonprofessional pop/rock musicians. Ear and Hearing. 2006;27(4):321-30.

14. Potier M, Hoquet C, Lloyd R, Nicolas-Puel C, Uziel A, Puel J-L. The risks of amplified music for disc jockeys working in nightclubs. Ear and Hearing. 2009; 30(2):291-3.

15. Steinmetz LG, Zeigelboim BS, Lacerda AB, Morata TC, Marques JM. Evaluating tinnitus in industrial hearing loss prevention programs. International Tinnitus Journal. 2008;14(2):152-8. 
16. Steinmetz LG, Zeigelboim BS; Lacerda AB; Morata TC; Marques JM. Características do zumbido em trabalhadores expostos a ruído. Revista Brasileira de Otorrinolaringologia. 2009;75(1):7-14. 17. Andrade AIA, Russo ICP, Lima MLLT, Oliveira LCS. Avaliação auditiva em músicos de frevo e maracatu. Revista Brasileira de Otorrinolaringologia. 2002; 68(5):714-20.

18. Mendes MH, Morata TC. Exposição ocupacional à música: uma revisão. Revista da Sociedade Brasileira de Fonoaudiologia. 2007;12(1):63-9.

19. Widén SE, Holmes AE, Erlandsson SI. Reported hearing protection use in young adults from Sweden and the USA: Effects of attitude and gender. International Journal of Audiology. 2006;45(5):273-80.

20. Gonçalves CGO, Couto CM, Carraro JM, Leonelli BS. Avaliação da colocação de protetores auriculares em grupos com e sem treinamento. Revista CEFAC. 2009;11(2):345-52.

21. Morata TC. Young people: Their noise and music exposures and the risk of hearing loss. International Journal of Audiology. 2007;46(3):111-2.
22. Mendes MH, Morata TC, Marques JM. Aceitação de protetores auditivos pelos componentes de banda instrumental e vocal. Revista Brasileira de Otorrinolaringologia. 2007;73(6):785-92.

23. Verbeek JH, Kateman E, Morata TC, Dreschler W, Sorgdrager B. Interventions to prevent occupational noise induced hearing loss. Cochrane Database of Systematic Reviews. 2009;3:1-69.

24. Zocoli AMF, Morata TC, Marques JM, Corteletti LJ. Brazilian young adults and noise: Attitudes, habits, and audiological characteristics. International Journal of Audiology. 2009;48(10):692-9.

25. Toppila E, Pyykko I, Starck J, Kaksonen R, Ishizaki $\mathrm{H}$. Individual risk factors in the development of noise-induced hearing loss. Noise and Health. 2006;2(8):59-70.

26. Pyykkö I, Toppila E, Zou J, Kentala E. Individual susceptibility to noise-induced hearing loss. Audiological Medicine. 2007;5(1):41-53.

27. Toppila E, Pyykko I, Starck J. Age and noiseinduced hearing loss. Scandinavian Audiology. 2008;30(4):236-44.

doi: 10.1590/S1516-18462010005000120

RECEBIDO EM: 10/02/2010

ACEITO EM: 20/07/2010

Endereço para correspondência:

Wagner Teobaldo Lopes de Andrade

Rua Marquês de Maricá, 236/202 - Torre

Recife/PE

CEP: 50711-120

E-mail: wagnerteobaldo @ccs.ufpb.br wagner_teobaldo@yahoo.com.br 